\title{
Green Synthesis and Characterization of Carbon Nanotubes/Polyaniline Nanocomposites
}

\author{
Van Hoa Nguyen ${ }^{1,2}$ and Jae-Jin Shim ${ }^{1}$ \\ ${ }^{1}$ School of Chemical Engineering, Yeungnam University, Gyeongsan, Gyeongbuk 712-749, Republic of Korea \\ ${ }^{2}$ Department of Chemistry, Nha Trang University, 2 Nguyen Dinh Chieu, Nha Trang, Vietnam \\ Correspondence should be addressed to Jae-Jin Shim; jjshim@yu.ac.kr
}

Received 16 October 2014; Revised 10 January 2015; Accepted 12 January 2015

Academic Editor: Dhananjay Bodas

Copyright (c) 2015 V. H. Nguyen and J.-J. Shim. This is an open access article distributed under the Creative Commons Attribution License, which permits unrestricted use, distribution, and reproduction in any medium, provided the original work is properly cited.

Carbon nanotubes/polyaniline (CNT/PANI) nanocomposites were synthesized by the interfacial polymerization of aniline in the presence of CNTs using two green solvents, water and an ionic liquid (1-butyl-3-methylimidazolium tetrafluoroborate, $\left.[\mathrm{bmim}]\left[\mathrm{BF}_{4}\right]\right)$, as the two phases. The formation and incorporation of PANI on the surface of the CNTs were confirmed by scanning electron microscopy, transmission electron microscopy, X-ray diffraction, Fourier transform infrared spectroscopy ultravioletvisible spectroscopy, and X-ray photoelectron spectroscopy. The analyses showed that the surface of the CNTs was coated with different morphologies of thin PANI layers depending on whether a $\mathrm{HCl}$ or $\mathrm{HNO}_{3}$ solution was used. The thermal stability of the composites was much better than that of the bare CNTs and pure PANI. The as-prepared composites were also used to modify the nickel foam electrodes for characterization of the electrochemical properties.

\section{Introduction}

Polyaniline (PANI) has attracted considerable attention because of its unique and controllable chemical and electrical properties; environmental, thermal, and electrochemical stability; and interesting electronic, optical, and electrooptical properties [1-4]. PANI has a wide range of tunable properties emanating from its structural flexibility, leading to potential applications in many fields, such as battery electrodes, anticorrosive coatings, energy storage systems, gas sensors, and electrocatalytic devices $[3,4]$. Moreover, PANI has the highest environmental stability and is recognized as the only conducting polymer stable in air [5]. However, PANI has low conductivity, which limits its electrochemical performance and applications in developing electrical devices. To overcome this problem, CNTs have been assessed as potential candidates owing to their unique structure and excellent mechanical, electrical, and thermal properties as well as their high surface area [6-8]. A method to prepare composites of the two materials is needed to combine the advantages of PANI and CNTs.
Composites consisting of CNTs and PANI have been developed for different applications in lithium ion batteries, supercapacitors, catalysts, solar cells, nanodevices, chemical sensors, and biosensors [6-10]. Improved stability of the composites has been achieved by the synergistic combination of the excellent conducting and mechanical properties of CNTs sheets with the high pseudo capacitance of PANI [9]. Moreover, a coating of CNTs with polyaniline enhances the conductivity and mechanical strength remarkably, resulting in excellent performance as an electrode material [10]. However, most of these studies normally require toxic organic solvents, which may be harmful to the environment. To address this, it is important to develop a facile and efficient method without toxic organic solvents. Green solvents, such as supercritical carbon dioxide and ionic liquids (ILs), should be developed to overcome these environmental concerns. ILs have unique properties, such as extremely low volatility, wide liquid temperature range, good thermal stability, good dissolving ability, designable structures, high ionic conductivity, wide electrochemical window, and excellent microwave 
absorbing ability [11, 12]. Previous studies successfully prepared polymers and nanocomposites in ILs [12-15].

In the present study, CNT/PANI nanocomposites were prepared by in situ interfacial polymerization using two green solvents, water and $[\mathrm{bmim}]\left[\mathrm{BF}_{4}\right]$. This method minimizes the use of toxic organic solvents. The products obtained were characterized using standard techniques. The composites were also used to modify the nickel foam electrodes for characterization of the electrochemical properties.

\section{Experimental}

2.1. Materials. MWNTs ( $>90 \%$, Aldrich) have a diameter and length of $10-50 \mathrm{~nm}$ and $0.1-10 \mu \mathrm{m}$, respectively. Aniline (99\%, Aldrich) was distilled under reduced pressure prior to use. $[\mathrm{Bmim}]\left[\mathrm{BF}_{4}\right]$ ( $>98 \%$, Ionic Liquids Tech., Germany) was kept in a vacuum oven at $100^{\circ} \mathrm{C}$ for $24 \mathrm{~h}$ to remove the volatile impurities prior to use. Potassium persulfate (KPS) and the other reagents were of analytical grade and used as received. Water was deionized in the laboratory. The carboxylic acid-functionalized CNTs (CNT-COOH) were obtained by sonication in a mixture of concentrated $\mathrm{H}_{2} \mathrm{SO}_{4}$ and $\mathrm{HNO}_{3}(3: 1, \mathrm{v} / \mathrm{v})$ at $50^{\circ} \mathrm{C}$ for 10 hours, followed by centrifugation of $15,000 \mathrm{rpm}$ and washing with deionized water until the decanted solution was neutral.

\subsection{Synthesis of CNT/PANI Composite. The CNT/PANI com-} posites were prepared using a methodology reported elsewhere [14]. In a typical experiment, $0.5 \mathrm{~g}$ aniline was dissolved in $10 \mathrm{~mL}$ IL, and $50 \mathrm{mg}$ CNT-COOH was dispersed in $10 \mathrm{~mL}$ DI water, which was followed by the addition of $0.5 \mathrm{~mL}$ hydrochloric acid $(1 \mathrm{M} \mathrm{HCl})$ or nitric acid $\left(1 \mathrm{M} \mathrm{HNO}_{3}\right)$ and $2.9 \mathrm{~g}$ of KPS. The KPS to aniline molar ratio was $2: 1$. The two solutions were transferred carefully to a vial, and an interface was formed between the aqueous phase and the IL phase. Green PANI that formed at the interface was then diffused gradually into the aqueous phase. After $3 \mathrm{~h}$, the entire water phase was filled homogeneously with a dark-green CNT/PANI nanocomposite. The aqueous phase was then collected and washed with ethanol and water to remove the unreacted chemicals and aniline oligomers. The CNT/PANI composite obtained was dried in a vacuum oven at $40^{\circ} \mathrm{C}$ for $24 \mathrm{~h}$.

2.3. Characterization. The samples were characterized by scanning electron microscopy (SEM, Hitachi, S-4200), transmission electron microscopy (TEM, Philips, CM-200) at an acceleration voltage of $200 \mathrm{kV}, \mathrm{X}$-ray diffraction (XRD, PANalytical, X'Pert-PRO MPD) using $\mathrm{Cu} K \alpha$ radiation, and X-ray photoelectron spectroscopy (XPS, ULVAC-PHI, Quantera SXM) using an Al X-ray source. The Fourier transform infrared (FTIR, Nicolet iS10, Thermo Scientific) spectra were recorded over $500-4000 \mathrm{~cm}^{-1}$ at a resolution of $16 \mathrm{~cm}^{-1}$ within 32 scans using a diamond ATR attachment. The ultraviolet-visible (UV-vis) spectra were recorded over 200$800 \mathrm{~nm}$ using a UV-Vis-NIR spectrophotometer (Cary 5000, Varian). Thermogravimetric analysis (TGA) was performed on a simultaneous TGA/differential scanning calorimetry
(DSC) analyzer (SDT Q600, TA Instrument) from 25 to $600^{\circ} \mathrm{C}$ at a heating rate of $10^{\circ} \mathrm{C} / \mathrm{min}$ under a nitrogen atmosphere.

All electrochemical experiments were performed on a VersaSTAT 3 AMETEK Model (Princeton Applied Research, USA) in a three-electrode configuration. Platinum foil and a saturated calomel electrode were used as the counter and reference electrodes, respectively. The working electrodes were fabricated by mixing the as-prepared powder $(3 \mathrm{mg}$, 80 wt. \%) with 15 wt. \% acetylene black and 5 wt. \% polytetrafluorene-ethylene (PTFE) binder and pressed onto nickel foam current-collectors $(1.0 \mathrm{~cm} \times 1.0 \mathrm{~cm})$. The measurements were carried out in a $1 \mathrm{M} \mathrm{KOH}$ aqueous electrolyte at room temperature. The specific capacitance $\left(C_{s}\right)$ of the electrode was calculated using the following equation:

$$
C=\frac{I t}{m \Delta V},
$$

where $C, I, t, m$, and $\Delta V$ are the specific capacitance $\left(\mathrm{Fg}^{-1}\right)$, discharging current $(\mathrm{A})$, discharging time $(\mathrm{s})$, mass of active materials $(\mathrm{g})$, and discharging potential range $(\mathrm{V})$, respectively.

\section{Results and Discussion}

Scheme 1 presents the preparation process of the CNT/PANI composites. The aniline monomer was dissolved in [bmim $]\left[\mathrm{BF}_{4}\right]$ and the functionalized CNTs were dispersed in an aqueous acid solution with the oxidant, KPS. The water layer was spread carefully onto an equal volume of IL, forming a water/IL interface. After approximately $1 \mathrm{~min}$, green PANI formed at the interface and migrated gradually to the aqueous phase. Finally, the entire water phase was filled homogeneously with a dark-green CNT/PANI composite, whereas the IL phase showed a red-orange color, possibly due to the formation of aniline oligomers. The composite was collected and purified. Scheme 1 also presents typical snapshots of the synthetic process of CNT/PANI composites in the water/IL system.

The surface morphology of the as-prepared composites was examined by SEM and TEM. Figure 1 presents SEM and TEM images of the CNT/PANI composites prepared in two different media, $\mathrm{HCl}$ and $\mathrm{HNO}_{3}$ aqueous solution. The surfaces of the CNTs were covered completely with PANI layers. On the other hand, the morphology of the PANI layers was different, nanosheet-like for the HCl medium and nanoparticle-like for the $\mathrm{HNO}_{3}$ medium. The crystal structure of conducting PANI has a considerable effect on its electrical conductivity. A previous study reported that strong electrical conductivity could be induced by the highly ordered chain structure of conducting polymers [16]. Figure 2 shows the XRD pattern of the modified CNTs, pure PANI, and CNT/PANI composites. The CNT spectrum has a very strong peak at $26.5^{\circ} 2 \theta$. Pure PANI showed a typical crystalline pattern, which was similar to that of the CNT/PANI composites. The main diffraction peaks at $21.26^{\circ}, 29.82^{\circ}$, and $30.89^{\circ}$ $2 \theta$ with $h k l$ values of (100), (211), and (020), respectively, represent emeraldine PANI [16]. The peaks centered at $21.26^{\circ}$ 


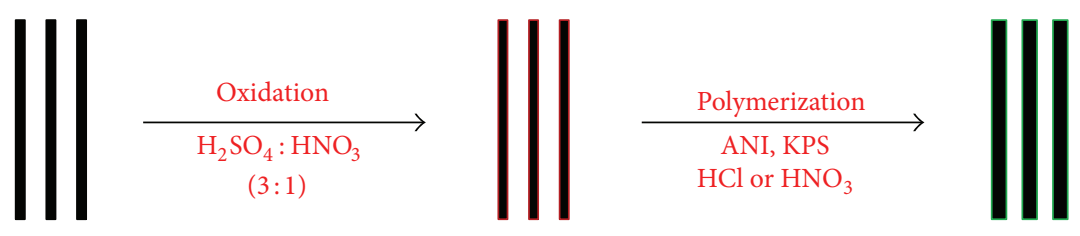

Bare CNTs

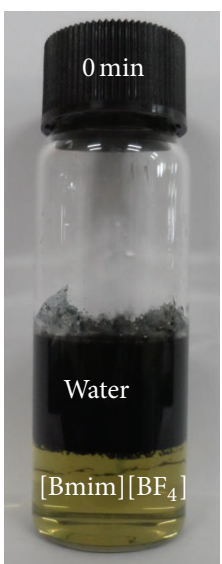

CNT-COOH

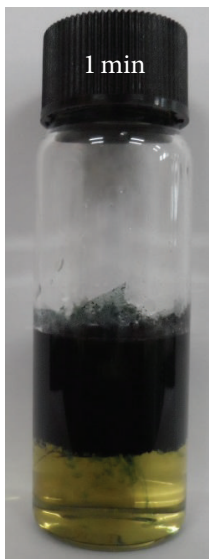

CNT/PANI

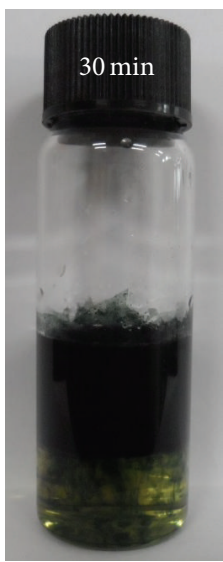

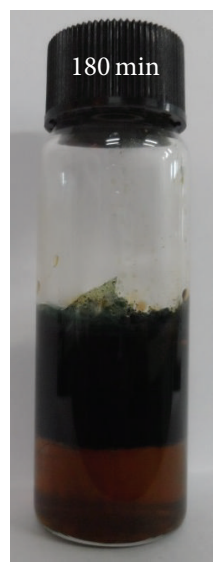

SCHEME 1: Schematic diagram of the synthesis of the CNT/PANI composite and the progress of the interfacial polymerization of aniline in a water/IL system in the presence of CNT.

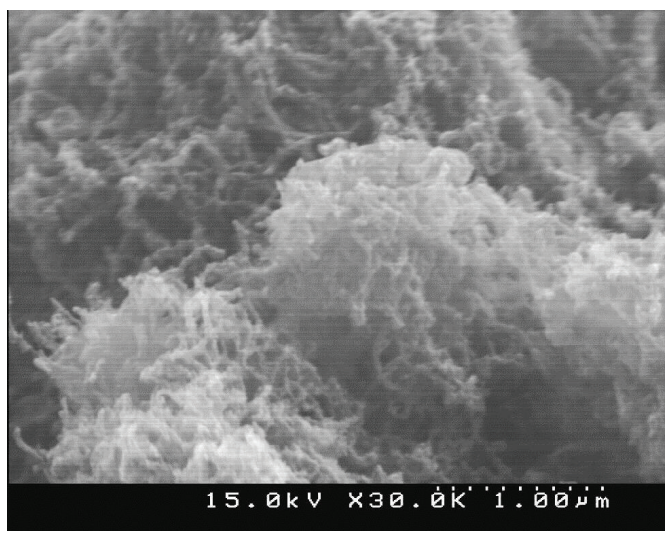

(a)

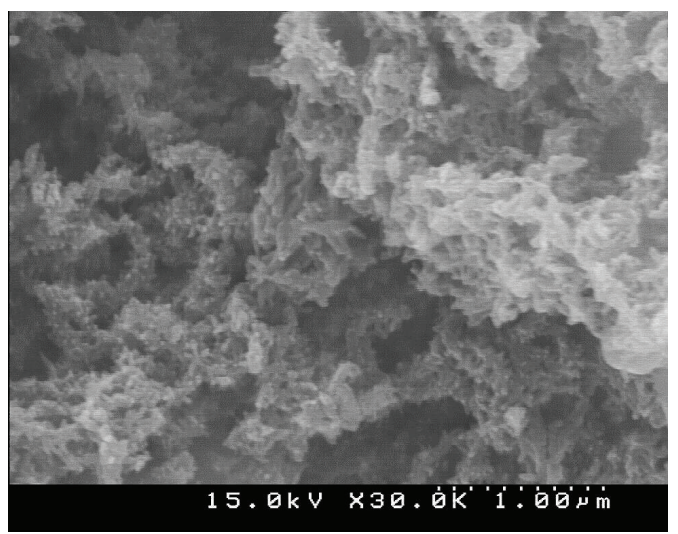

(c)

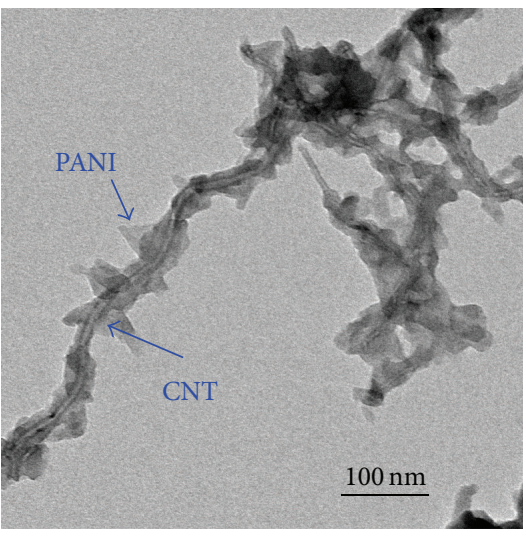

(b)

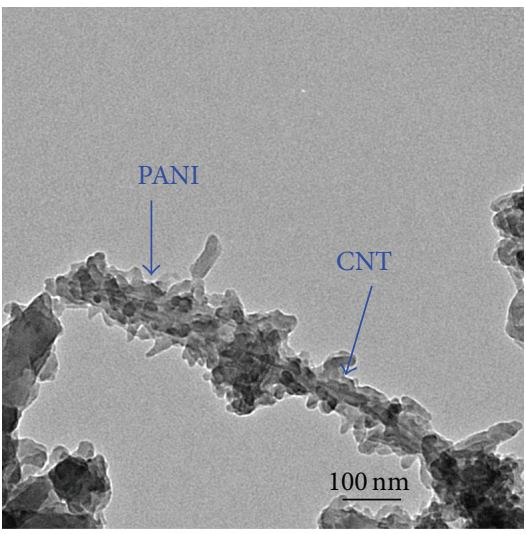

(d)

Figure 1: SEM (a, c) and TEM (b, d) images of CNT/PANI composites prepared in $\mathrm{HCl}(\mathrm{a}, \mathrm{b})$ and $\mathrm{HNO}_{3}(\mathrm{c}$, d) medium. 


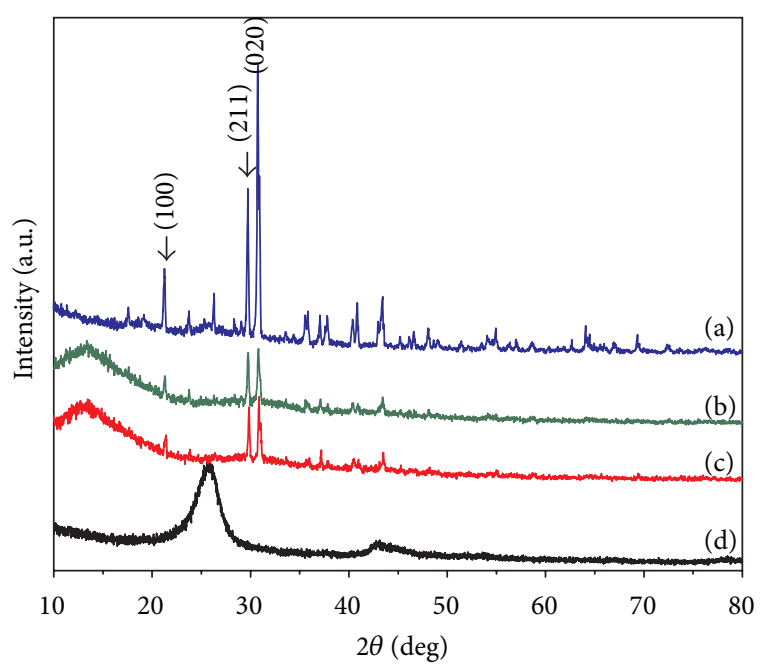

FIGURE 2: XRD patterns of pure PANI (a), CNT/PANI composites prepared in $\mathrm{HCl}$ (b) and $\mathrm{HNO}_{3}$ (c) medium, and $\mathrm{CNT}-\mathrm{COOH}$ (d).

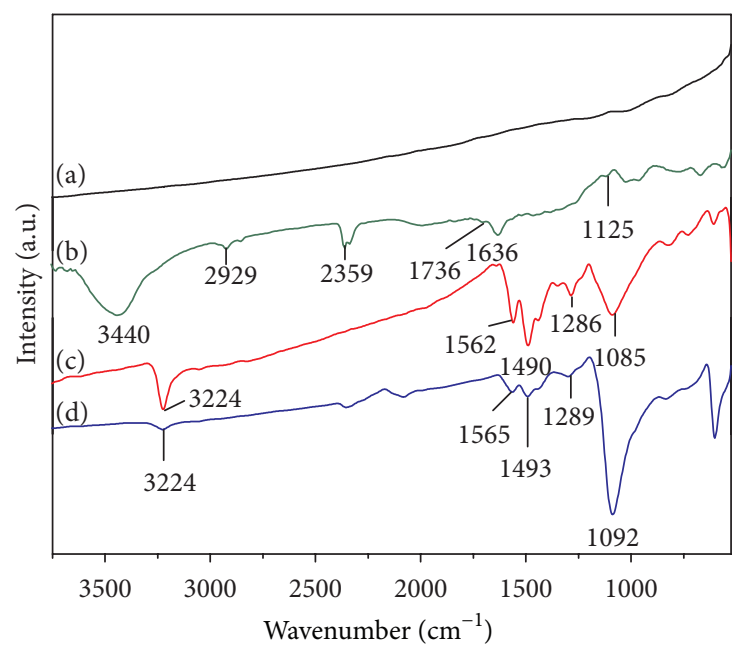

FIgURE 3: FTIR spectra of bare CNTs (a), CNT-COOH (b), pure PANI (c), and CNT/PANI composites prepared in $\mathrm{HCl}$ medium (d).

and $23.83^{\circ} 2 \theta$ were assigned to the periodicity parallel and perpendicular, respectively, to the PANI chains. XRD showed that PANI has a relatively highly ordered crystal structure, which improves the crystallinity of the composite considerably, and is expected to exhibit high electrical conductivity. The decrease in peak height of the CNT planes and the shift to the left in the composite might be caused by the polymer coating of the CNTs surface in the polymerization process.

FTIR spectroscopy provides information on the chemical structures of the bare CNTs, CNT-COOH, pure PANI, and CNT/PANI composites (Figure 3). The spectrum of bare CNTs showed no clear peaks at $500-3800 \mathrm{~cm}^{-1}$ (Figure 3(a)). The FTIR spectrum from CNT- $\mathrm{COOH}$ showed a broad peak at about $3440 \mathrm{~cm}^{-1}$ which could be assigned to the $\mathrm{O}-\mathrm{H}$ stretch from carboxyl groups $(\mathrm{O}=\mathrm{C}-\mathrm{OH}$ and $\mathrm{C}-\mathrm{OH})$ while the peak at $2359 \mathrm{~cm}^{-1}$ can be associated with the $\mathrm{O}-\mathrm{H}$ stretch from strongly hydrogen-bonded $-\mathrm{COOH}$ (Figure 3(b)) [17].

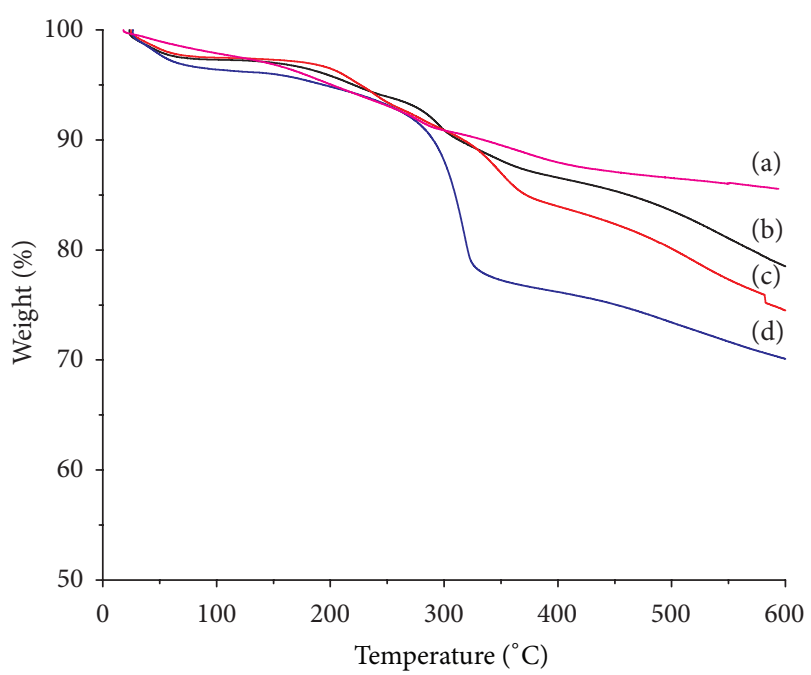

FIGURE 4: TGA thermograms of CNT-COOH (a), CNT/PANI composite prepared in $\mathrm{HCl}$ (b) and $\mathrm{HNO}_{3}$ (c) medium, and pure PANI (d), obtained with a ramping rate of $10^{\circ} \mathrm{C} / \mathrm{min}$ under a $\mathrm{N}_{2}$ atmosphere.

Moreover, the curve showed the characteristic $\mathrm{C}=\mathrm{C}$ stretching at $1634 \mathrm{~cm}^{-1}$, while $\mathrm{C}=\mathrm{O}$ and $\mathrm{C}-\mathrm{O}$ stretching vibrations are observed at 1713 and $1125 \mathrm{~cm}^{-1}$, respectively. The spectrum of pure PANI showed usual characteristic stretching vibration bands at $1562 \mathrm{~cm}^{-1}$ ( $\mathrm{C}=\mathrm{C}$, quinoid rings), $1490 \mathrm{~cm}^{-1}(\mathrm{C}=\mathrm{C}$, benzenoid rings), $1286 \mathrm{~cm}^{-1}(\mathrm{C}-\mathrm{N})$, and $1085 \mathrm{~cm}^{-1}$ (C$\mathrm{H})$ (Figure 3(c)). In the case of the CNT/PANI composite (Figure 3(d)), the FTIR spectrum was similar to that of PANI. These results confirmed that the surface of the CNTs was wrapped completely with PANI.

Figure 4 shows the TGA data of the modified CNTs, pure PANI, and CNT/PANI composites under a nitrogen atmosphere. All the samples showed similar decomposition curves. On the other hand, pure PANI had much lower thermal stability than the CNTs and CNT/PANI composite. The gradual weight loss of PANI between 100 and $280^{\circ} \mathrm{C}$ was attributed to the deprotonation of PANI through the loss of dopant $\mathrm{HCl}$. The major weight loss $(\sim 30 \%)$ before $600^{\circ} \mathrm{C}$ was assigned to the degradation and decomposition of PANI with different degrees of polymerization. The weight reduction of CNTs observed at temperatures less than $100^{\circ} \mathrm{C}$ and between 150 and $300^{\circ} \mathrm{C}$ was consistent with the degradation of absorbed water and oxygen functional groups, respectively. These $-\mathrm{COOH}$ groups on the walls of the CNTs not only enhanced the dispersibility but also could anchor PANI to the surface. For the composite, the gradual degradation below $280^{\circ} \mathrm{C}$ was attributed to the evaporation of absorbed solvent and the decomposition of oxygen groups on the CNT surface. The weight loss at approximately $300^{\circ} \mathrm{C}$ was consistent with the degradation and decomposition of PANI. TGA suggested that the CNT/PANI composite has higher and slightly lower thermal stability pure PANI. This might be because of the strong interactions between CNT and PANI, which impose a restriction on the decomposition of the $-\mathrm{COOH}$ groups remaining on the CNT surface. These interactions can result 


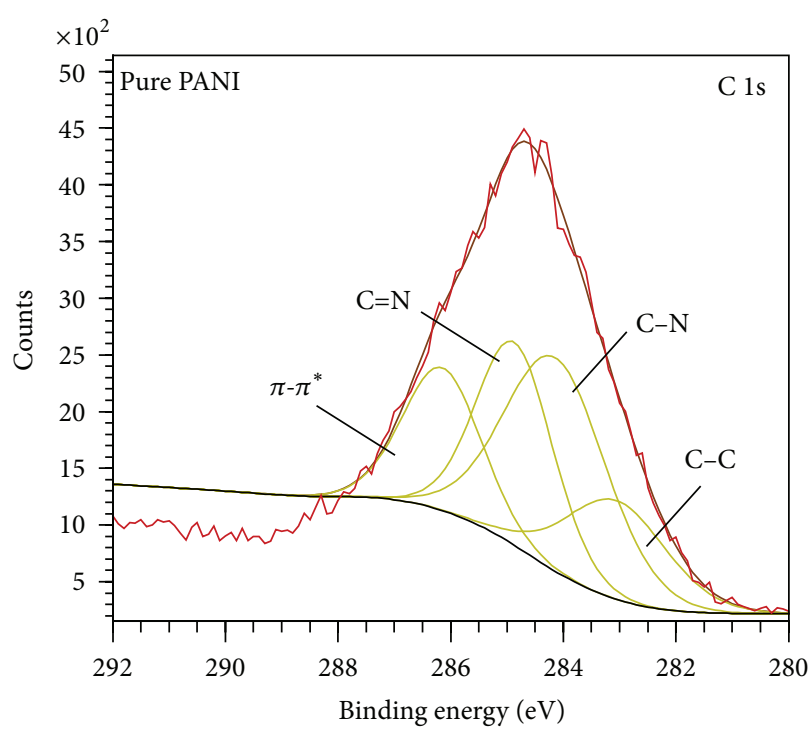

(a)

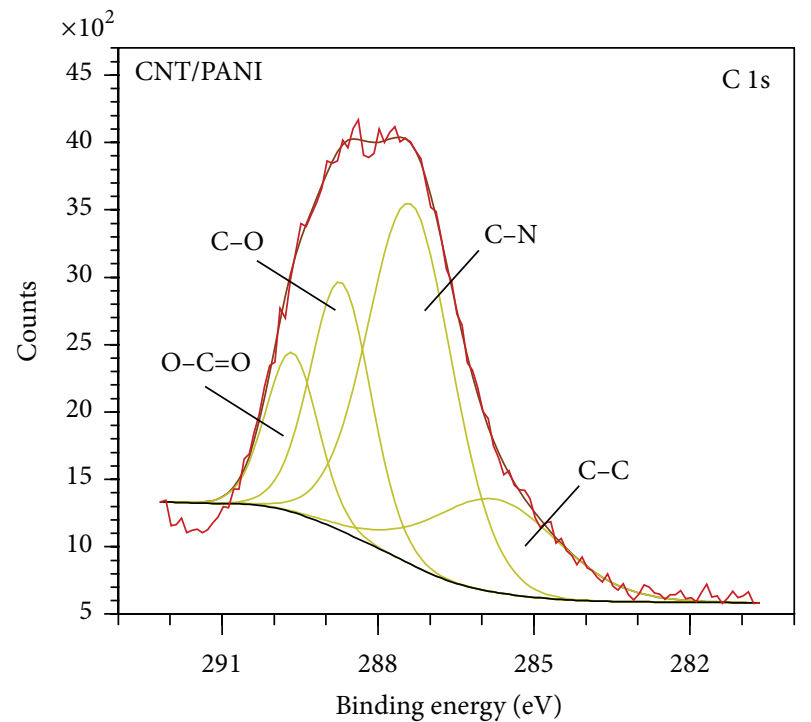

(c)

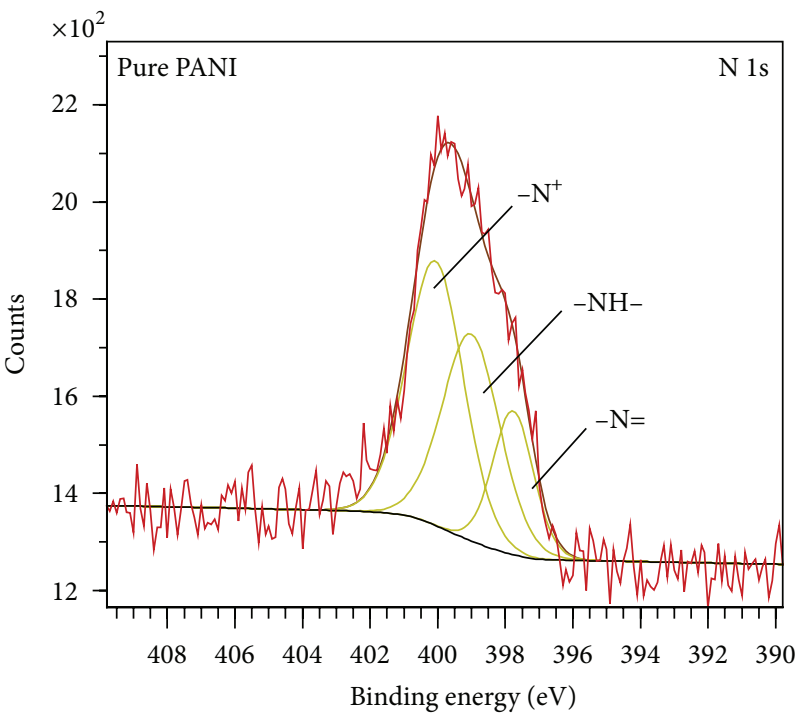

(b)

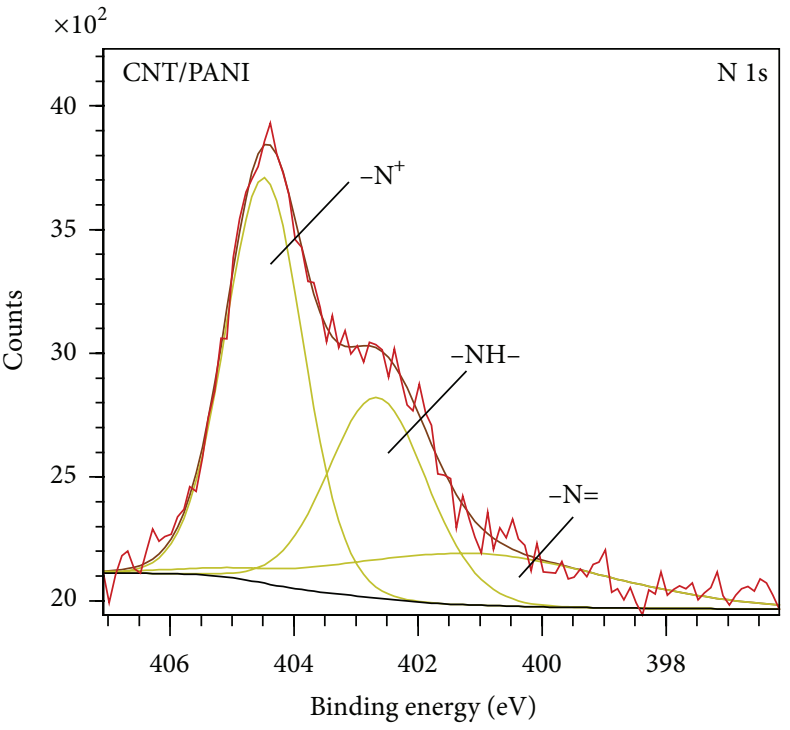

(d)

FIGURE 5: XPS survey spectra of pure PANI and the CNT/PANI composites prepared in $\mathrm{HCl}$ medium at core-levels of C 1s and N 1 s.

in homogeneous heating and the avoidance of heat concentrations [18].

Figure 5 shows the XPS spectrum of the N 1s core level of pure PANI, which is composed of three subpeaks centered at $397.8 \mathrm{eV}(-\mathrm{N}=), 399.0 \mathrm{eV}(-\mathrm{NH}-)$, and $400.1 \mathrm{eV}\left(-\mathrm{N}^{+}-\right)$. When combined with CNTs, the $\mathrm{N}^{+}$peak $(404.5 \mathrm{eV})$ of the CNT-PANI composite had a higher binding energy than pure PANI. The highest binding energy peak was assigned to the protonated amine units. Figure 5 also shows the $\mathrm{C}$ 1s spectrum of pure PANI. The lowest binding energy feature $(283.1 \mathrm{eV})$ was assigned to the $\mathrm{C}-\mathrm{C}$ group in the aromatic ring. The second peak was attributed to the $\mathrm{C}-\mathrm{N}$ bonds $(284.1 \mathrm{eV})$. The feature at $284.9 \mathrm{eV}$ can be attributed to $\mathrm{C}=\mathrm{N}$. An additional component centered at $286.2 \mathrm{eV}$ was attributed to the $\pi-\pi^{*}$ "shake-up" satellite. Compared to the C $1 \mathrm{~s}$ spectrum of the CNT-PANI composite, these C 1s spectra showed asymmetric characteristics, indicating the presence of structural defects. These $\mathrm{C}$ 1s spectra can be separated into four peaks, which were centered at approximately 285.7 (CC), $287.4(\mathrm{C}-\mathrm{N}), 288.7(\mathrm{C}-\mathrm{O})$, and $289.7 \mathrm{eV}(\mathrm{O}-\mathrm{C}=\mathrm{O})$. As a result, XPS showed that the CNT-PANI composites had been doped successfully with CNT, which may result in better electrochemical properties of the composite.

Figure 6 presents the UV-vis absorption spectra of pure PANI, functionalized CNT, and CNT/PANI dispersed in ethanol. Dispersed individual CNTs are active in the UVvis region and exhibit characteristic bands at approximately $250 \mathrm{~nm}$ corresponding to additional absorption due to the $1 \mathrm{D}$ van Hove singularities [19] (Figure 5(a)). The PANI spectrum revealed a sharp peak at $209 \mathrm{~nm}$, which was assigned to 


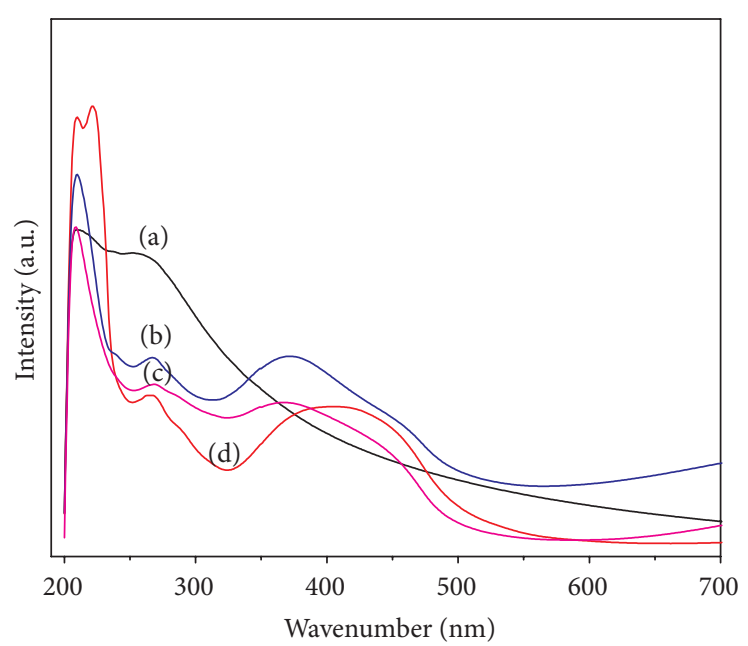

FIGURE 6: UV-visible spectra of the CNT-COOH (a), CNT/PANI composite prepared in $\mathrm{HCl}$ (b) and $\mathrm{HNO}_{3}$ (c) medium, and pure PANI (d) in ethanol.

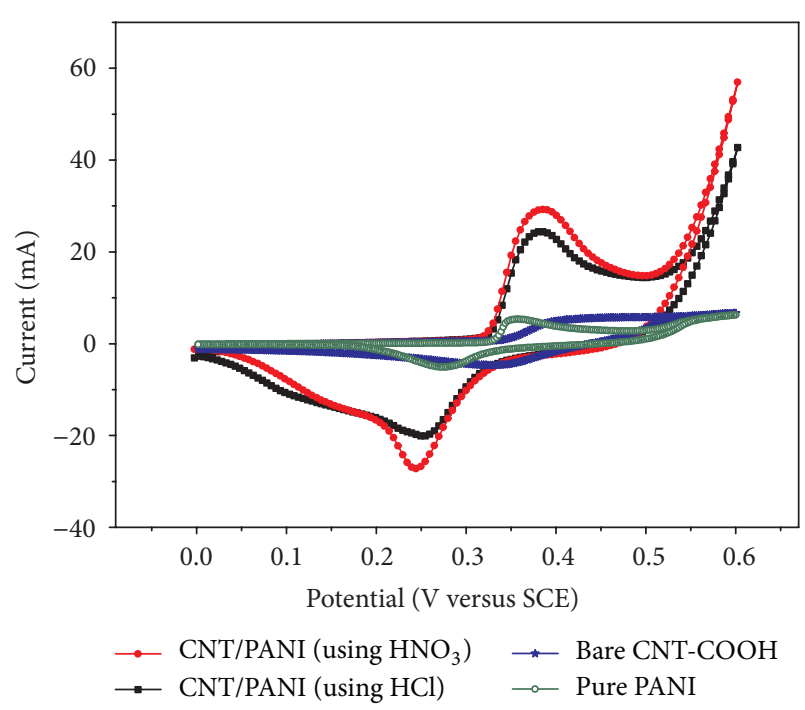

(a)

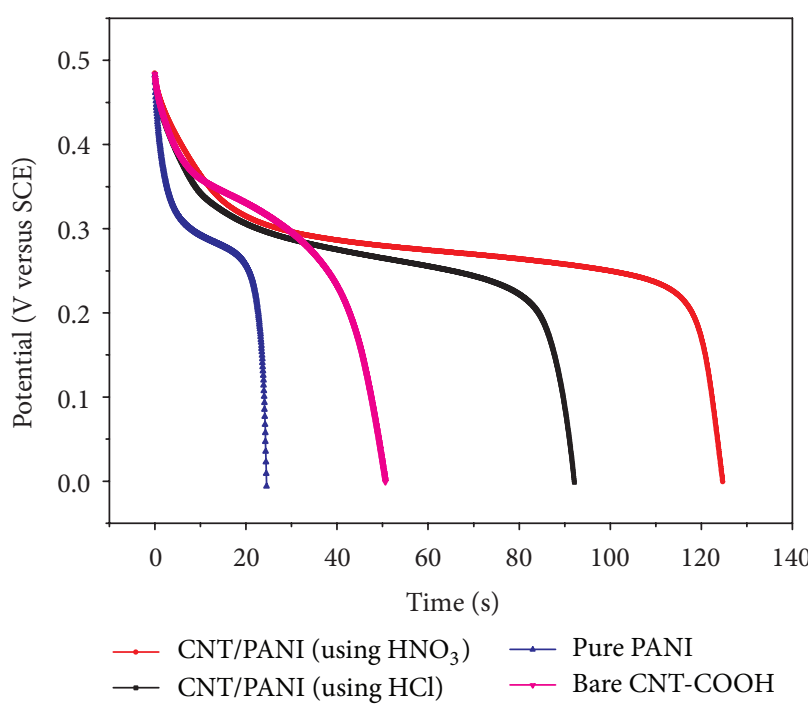

(b)

Figure 7: (a) CV curves of pure PANI, bare CNT-COOH, and CNT/PANI composites at scan rate of $10 \mathrm{~V} \mathrm{~s}^{-1}$; (b) galvanostatic discharge curves of pure PANI, bare CNT-COOH, and CNT/PANI composites at a current density of $2 \mathrm{Ag} \mathrm{g}^{-1}$.

the $\pi-\pi^{*}$ transition in molecular conjugation. A weak peak at $265 \mathrm{~nm}$ and broad peak at approximately $415 \mathrm{~nm}$ originated from the charged cationic species, called polarons [20]. For the CNT/PANI composites prepared in both $\mathrm{HCl}$ and $\mathrm{HNO}_{3}$ media, two peaks were observed at 207 and $268 \mathrm{~nm}$ due to a $\pi-\pi$ interaction between the PANI chains and CNTs in the composite. Compared to PANI, the peak shift from $415 \mathrm{~nm}$ to $372 \mathrm{~nm}$ indicated that PANI is protonated in the synthesized composite. The UV-vis absorption results confirmed the strong interaction between the PANI polymer and carbon tubes.

The electrochemical performance of the CNT/PANI composites as active supercapacitor electrodes was examined by cyclic voltammetry (CV), electrochemical impedance spectroscopy (EIS), and chronopotentiometry (CP) carried out in a three-electrode system using a SCE and platinum foil as the reference and counter electrode (Figures 7 and 8). At the same scan rate of $50 \mathrm{mV} \mathrm{s}^{-1}$ (Figure 7(a)), the CNT/PANI composites exhibited a much higher resulting current and a more rectangular shape than those of the bare CNT$\mathrm{COOH}$ and the pure PANI. Interestingly, the CNT/PANI (prepared in a $\mathrm{HNO}_{3}$ solution) electrode exhibited a much higher current than those of the CNT/PANI (prepared in a $\mathrm{HCl}$ solution) electrode, suggesting that the composite doped with $\mathrm{H}^{+}$from $\mathrm{HNO}_{3}$ had higher electron transport (Figures 8(a) and 8(b)). The improved electrochemical 

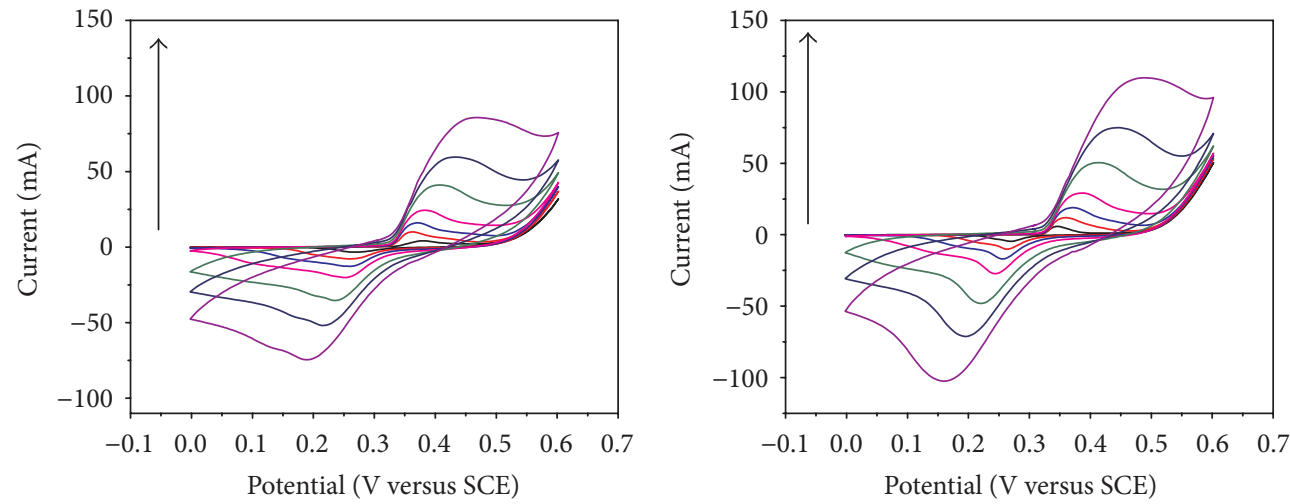

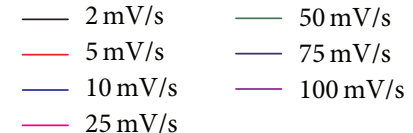

(a)
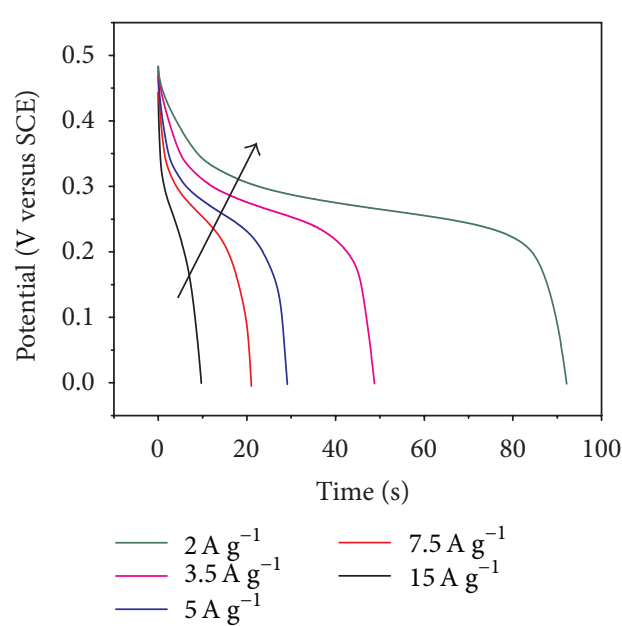

(c)

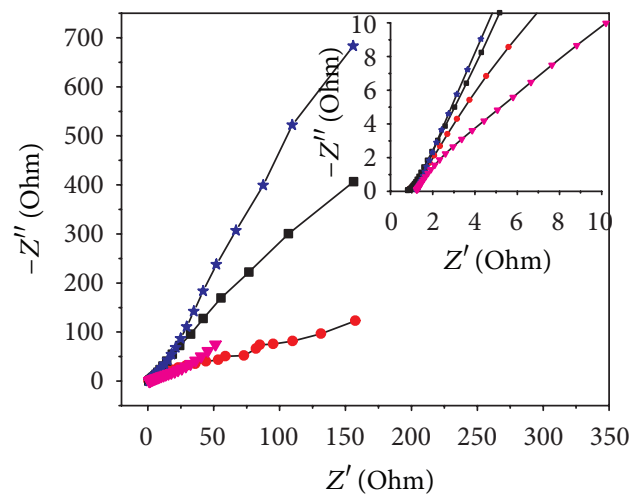

$\rightarrow$ Bare CNT-COOH using $\mathrm{HNO}_{3}$

CNT/PANI

(using $\mathrm{HCl}$ )

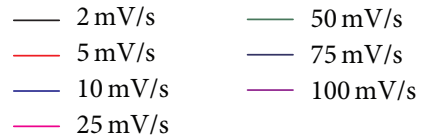

(b)

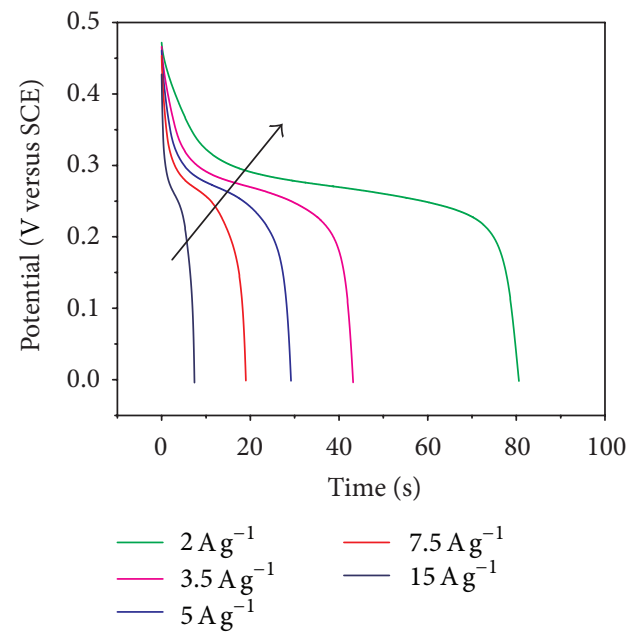

(d)

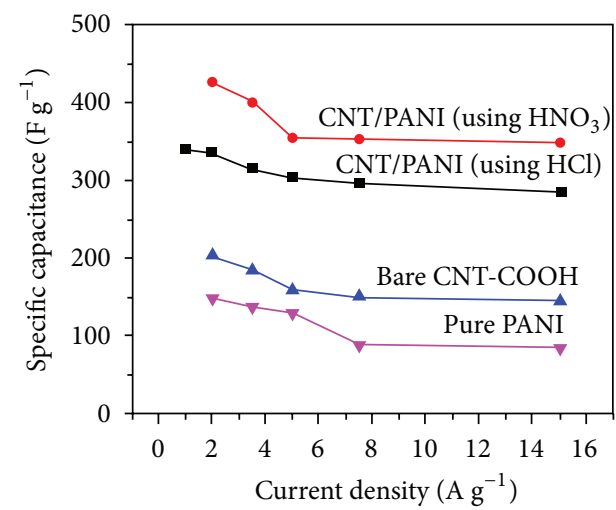

(e)

(f)

Figure 8: CV curves of the CNT/PANI composite prepared in $\mathrm{HCl}$ (a) and $\mathrm{HNO}_{3}$ (b) in a $1 \mathrm{M} \mathrm{KOH}$ solution at different scan rates; galvanostatic discharge curves of the CNT/PANI composites prepared in $\mathrm{HCl}$ (a) and $\mathrm{HNO}_{3}$ (b) at different current densities; (e) Nyquist plots of the pure PANI, bare CNT-COOH, and CNT/PANI composites; (f) the specific capacitance of the pure PANI, bare CNT-COOH, and $\mathrm{CNT} / \mathrm{PANI}$ composite electrodes at different current densities. 
performance was also confirmed by galvanostatic chargedischarge tests performed under the same current density for pure PANI, bare CNT-COOH, and CNT/PANI (Figure 7(b)) and at different current densities (Figures $8(\mathrm{c})$ and $8(\mathrm{~d})$ ). In particular, two composite electrodes showed excellent rate performance in comparison to that of pure PANI and bare CNT-COOH. Moreover, a pair of smaller platforms with the starting and ending potentials close to those in the CV curves (Figures $8(\mathrm{a})$ and $8(\mathrm{~b})$ ) are shown in the discharge curves (Figures $8(\mathrm{c})$ and $8(\mathrm{~d})$ ), which is a typical characteristic of the existence of redox reactions during the charge-discharge process. In addition, all discharge curves showed a very small IR drop, which indicated the low internal resistance of the electrodes. Figure 8(e) presents the impedance curves for the pure PANI, bare CNT-COOH, and CNT/PANI electrodes measured in a $1 \mathrm{M} \mathrm{KOH}$ electrolyte solution. The more vertical line in the low and high frequency regions indicated the more capacitive behavior of the electrodes. Figure 8(f) summarizes the specific capacitance at different current densities calculated from the discharge curves. The CNT/PANI composites yielded much enhanced capacitance performance compared to that of the pure PANI and bare CNT-COOH. In the other hand, the CNT/PANI (prepared in $\mathrm{HNO}_{3}$ solution) electrode exhibited enhanced capacitance performance with an approximate $8 \%$ increase in specific capacitance compared to that of CNT/PANI (prepared in $\mathrm{HCl}$ solution) electrode. What is more, the specific capacitance obtained in our work is considerable compared with the other morphologies that have been reported in literatures, such as SWCNT/PANI (350-485 $\left.\mathrm{Fg}^{-1}\right)$ [21-23], porous carbon/PANI (160-180 $\left.\mathrm{F} \mathrm{g}^{-1}\right)$ [24, 25], MWCNT/PANI (322$\left.606 \mathrm{Fg}^{-1}\right)$ [26-29], activated carbon/PANI (380-500 $\left.\mathrm{Fg}^{-1}\right)$ $[30,31]$, and carbon nanofiber/PANI $\left(264 \mathrm{~F} \mathrm{~g}^{-1}\right)$ [32].

\section{Conclusions}

CNT/PANI composites were prepared efficiently in an ILwater system via the typical interfacial polymerization of an aniline monomer. XRD confirmed the highly ordered chain structure of pure PANI and PANI in the composite. SEM and TEM indicated that the polymer layer almost completely covered the carbon tubes. The resulting CNT/PAN composite showed higher thermal stability than pure PANI. The electrochemical performance of the as-prepared CNT/PANI composites as active supercapacitor material electrodes was evaluated. The CNT/PANI (prepared in $\mathrm{HNO}_{3}$ solution) electrode showed enhanced capacitance performance with an approximate $8 \%$ increase in specific capacitance compared to that of the CNT/PANI (prepared in a $\mathrm{HCl}$ solution) electrode. This experimental method is a facile, efficient, and green route that can be used to develop other PANI-based composites.

\section{Conflict of Interests}

The authors declare that there is no conflict of interests regarding the publication of this paper.

\section{Acknowledgment}

This research was supported by the Priority Research Centers Program through the National Research Foundation of Korea (NRF) funded by the Ministry of Education (2014R1A6A1031189).

\section{References}

[1] A. P. Alivisatos, "Semiconductor clusters, nanocrystals, and quantum dots," Science, vol. 271, no. 5251, pp. 933-937, 1996.

[2] C. Dhand, M. Das, M. Datta, and B. D. Malhotra, "Recent advances in polyaniline based biosensors," Biosensors and Bioelectronics, vol. 26, no. 6, pp. 2811-2821, 2011.

[3] S. Bhadra, D. Khastgir, N. K. Singha, and J. H. Lee, "Progress in preparation, processing and applications of polyaniline," Progress in Polymer Science, vol. 34, no. 8, pp. 783-810, 2009.

[4] T. A. Sergeyeva, N. V. Lavrik, S. A. Piletsky, A. E. Rachkov, and A. V. El'skaya, "Polyaniline label-based conductometric sensor for IgG detection," Sensors and Actuators, B: Chemical, vol. 34, no. 1-3, pp. 283-288, 1996.

[5] Y. C. Luo and J. S. Do, "Urea biosensor based on PANi(urease)Nafion/Au composite electrode," Biosensors and Bioelectronics, vol. 20, no. 1, pp. 15-23, 2004.

[6] V. H. Nguyen and J.-J. Shim, "Facile synthesis and characterization of carbon nanotubes/silver nanohybrids coated with polyaniline," Synthetic Metals, vol. 161, no. 19-20, pp. 2078-2082, 2011.

[7] V. H. Nguyen and J.-J. Shima, "Supercritical fluid-assisted synthesis of a carbon nanotubes-grafted biocompatible polymer composite," Composite Interfaces, vol. 20, no. 2, pp. 155-162, 2013.

[8] M. S. Shamsudin, A. B. Suriani, S. Abdullah, S. Y. S. Yahya, and M. Rusop, "Impact of thermal annealing under nitrogen ambient on structural, micro-Raman, and thermogravimetric analyses of camphoric-CNT," Journal of Spectroscopy, vol. 2013, Article ID 167357, 6 pages, 2013.

[9] C. Oueiny, S. Berlioz, and F.-X. Perrin, "Carbon nanotubepolyaniline composites," Progress in Polymer Science, vol. 39, no. 4, pp. 707-748, 2014.

[10] P. Gajendran and R. Saraswathi, "Polyaniline-carbon nanotube composites," Pure and Applied Chemistry, vol. 80, no. 11, pp. 2377-2395, 2008.

[11] J.-H. Pang, X. Liu, M. Wu, Y.-Y. Wu, X.-M. Zhang, and R.-C. Sun, "Fabrication and characterization of regenerated cellulose films using different ionic liquids," Journal of Spectroscopy, vol. 2014, Article ID 214057, 8 pages, 2014.

[12] V. H. Nguyen, Y. P. Ren, Y. R. Lee, D. Tuma, B. K. Min, and J. J. Shim, "Microwave-assisted synthesis of carbon nanotube$\mathrm{TiO}_{2}$ nanocomposites in ionic liquid for the photocatalytic degradation of methylene blue," Synthesis and Reactivity in Inorganic, Metal-Organic, and Nano-Metal Chemistry, vol. 42, no. 2, pp. 296-301, 2012.

[13] V. H. Nguyen, Y. Haldorai, Q. L. Pham, S. K. Noh, W. S. Lyoo, and J.-J. Shim, "Preparation of poly(vinyl pivalate) microspheres by dispersion polymerization in an ionic liquid and saponification for the preparation of poly(vinyl alcohol) with high syndiotacticity," European Polymer Journal, vol. 46, no. 11, pp. 2190-2198, 2010.

[14] V. H. Nguyen, L. Tang, and J.-J. Shim, "Electrochemical property of graphene oxide/polyaniline composite prepared by in 
situ interfacial polymerization," Colloid and Polymer Science, vol. 291, no. 9, pp. 2237-2243, 2013.

[15] V. H. Nguyen and J.-J. Shim, "Ionic liquid mediated synthesis of graphene- $\mathrm{TiO}_{2}$ hybrid and its photocatalytic activity," Materials Science and Engineering B, vol. 180, no. 1, pp. 38-45, 2014.

[16] J. P. Pouget, M. E. Jozefowicz, A. J. Epstein, X. Tang, and A. G. MacDiarmid, "X-ray structure of polyaniline," Macromolecules, vol. 24, no. 3, pp. 779-789, 1991.

[17] M. A. Atieh, O. Y. Bakather, B. Al-Tawbini, A. A. Bukhari, F. A. Abuilaiwi, and M. B. Fettouhi, "Effect of carboxylic functional group functionalized on carbon nanotubes surface on the removal of lead from water," Bioinorganic Chemistry and Applications, vol. 2010, Article ID 603978, 9 pages, 2010.

[18] H. Wang, Q. Hao, X. Yang, L. Lu, and X. Wang, "Effect of graphene oxide on the properties of its composite with polyaniline," ACS Applied Materials and Interfaces, vol. 2, no. 3, pp. 821-828, 2010.

[19] J. Yu, N. Grossiord, C. E. Koning, and J. Loos, "Controlling the dispersion of multi-wall carbon nanotubes in aqueous surfactant solution," Carbon, vol. 45, no. 3, pp. 618-623, 2007.

[20] J. Stejskal, P. Kratochvíl, and N. Radhakrishnan, "Polyaniline dispersions 2. UV-Vis absorption spectra," Synthetic Metals, vol. 61, no. 3, pp. 225-231, 1993.

[21] V. Gupta and N. Miura, "Polyaniline/single-wall carbon nanotube (PANI/SWCNT) composites for high performance supercapacitors," Electrochimica Acta, vol. 52, no. 4, pp. 17211726, 2006.

[22] V. Gupta and N. Miura, "Influence of the microstructure on the supercapacitive behavior of polyaniline/single-wall carbon nanotube composites," Journal of Power Sources, vol. 157, no. 1, pp. 616-620, 2006.

[23] Y.-K. Zhou, B.-L. He, W.-J. Zhou, and H.-L. Li, "Preparation and electrochemistry of SWNT/PANI composite films for electrochemical capacitors," Journal of the Electrochemical Society, vol. 151, no. 7, pp. A1052-A1057, 2004.

[24] W.-C. Chen and T.-C. Wen, "Electrochemical and capacitive properties of polyaniline-implanted porous carbon electrode for supercapacitors," Journal of Power Sources, vol. 117, no. 1-2, pp. 273-282, 2003.

[25] W.-C. Chen, T.-C. Wen, and H. S. Teng, "Polyaniline-deposited porous carbon electrode for supercapacitor," Electrochimica Acta, vol. 48, no. 6, pp. 641-649, 2003.

[26] S. R. Sivakkumar, W. J. Kim, J.-A. Choi, D. R. MacFarlane, M. Forsyth, and D.-W. Kim, "Electrochemical performance of polyaniline nanofibres and polyaniline/multi-walled carbon nanotube composite as an electrode material for aqueous redox supercapacitors," Journal of Power Sources, vol. 171, no. 2, pp. 1062-1068, 2007.

[27] H. Y. Mi, X. G. Zhang, S. Y. An, X. G. Ye, and S. D. Yang, "Microwave-assisted synthesis and electrochemical capacitance of polyaniline/multi-wall carbon nanotubes composite," Electrochemistry Communications, vol. 9, no. 12, pp. 2859-2862, 2007.

[28] Y. Zhou, Z.-Y. Qin, L. Li et al., "Polyaniline/multi-walled carbon nanotube composites with core-shell structures as supercapacitor electrode materials," Electrochimica Acta, vol. 55, no. 12, pp. 3904-3908, 2010.

[29] B. Dong, B.-L. He, C.-L. Xu, and H.-L. Li, "Preparation and electrochemical characterization of polyaniline/multi-walled carbon nanotubes composites for supercapacitor," Materials Science and Engineering B: Solid-State Materials for Advanced Technology, vol. 143, no. 1-3, pp. 7-13, 2007.
[30] J. H. Park and O. O. Park, "Hybrid electrochemical capacitors based on polyaniline and activated carbon electrodes," Journal of Power Sources, vol. 111, no. 1, pp. 185-190, 2002.

[31] M. J. Bleda-Martínez, C. Peng, S. Zhang, G. Z. Chen, E. Morallón, and D. Cazorla-Amorós, "Electrochemical methods to enhance the capacitance in activated carbon/polyaniline composites," Journal of the Electrochemical Society, vol. 155, no. 9, pp. A672-A678, 2008.

[32] J. Jang, J. Bae, M. Choi, and S.-H. Yoon, "Fabrication and characterization of polyaniline coated carbon nanofiber for supercapacitor," Carbon, vol. 43, no. 13, pp. 2730-2736, 2005. 

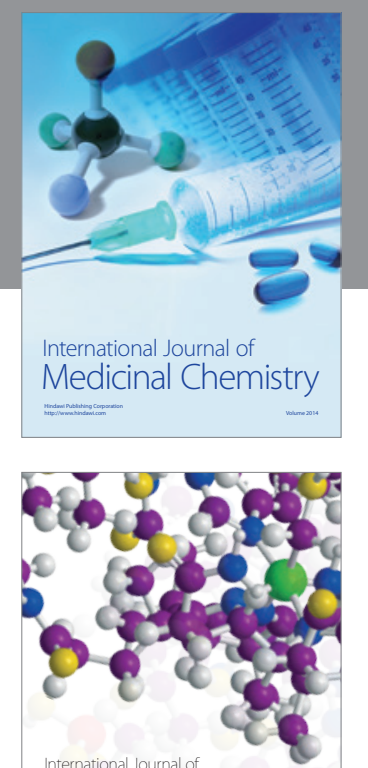

\section{Carbohydrate} Chemistry

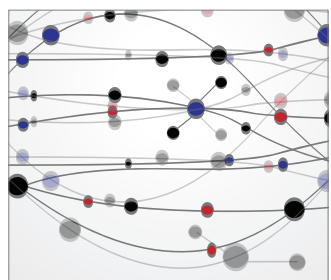

The Scientific World Journal
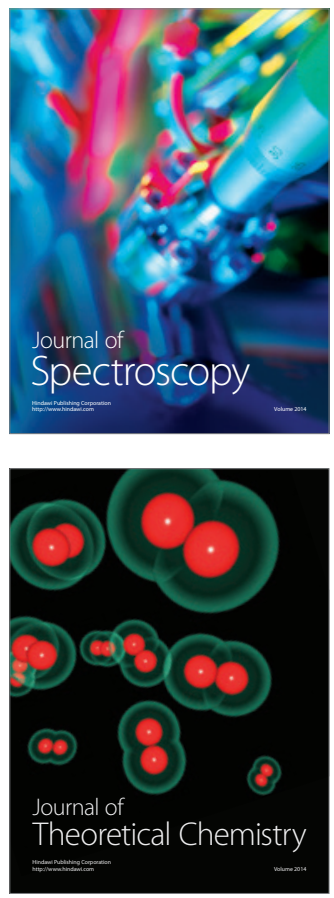
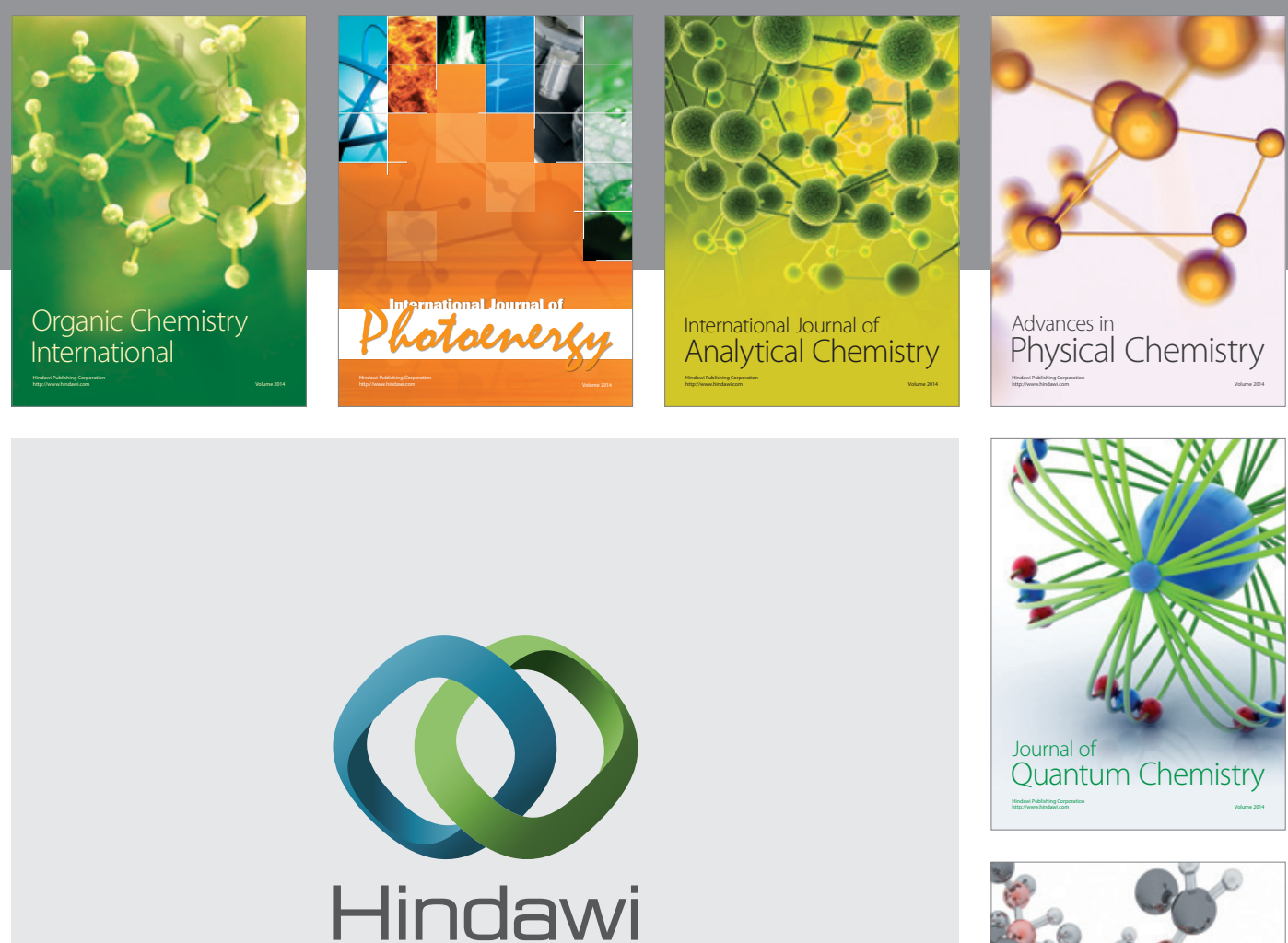

Submit your manuscripts at

http://www.hindawi.com

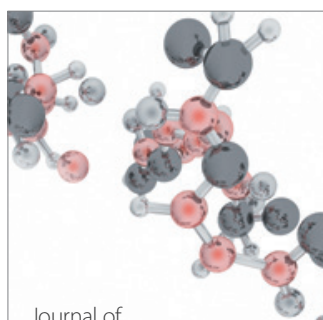

Analytical Methods

in Chemistry

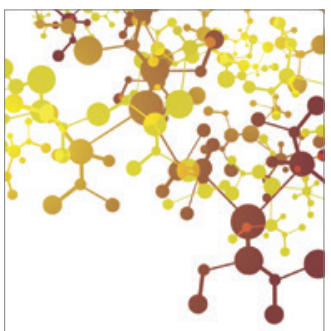

Journal of

Applied Chemistry

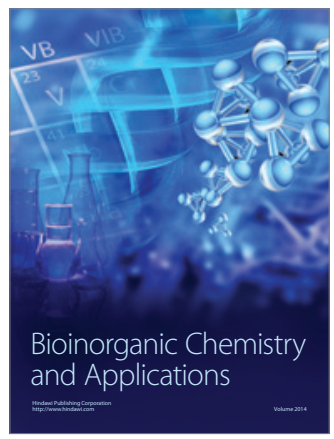

Inorganic Chemistry
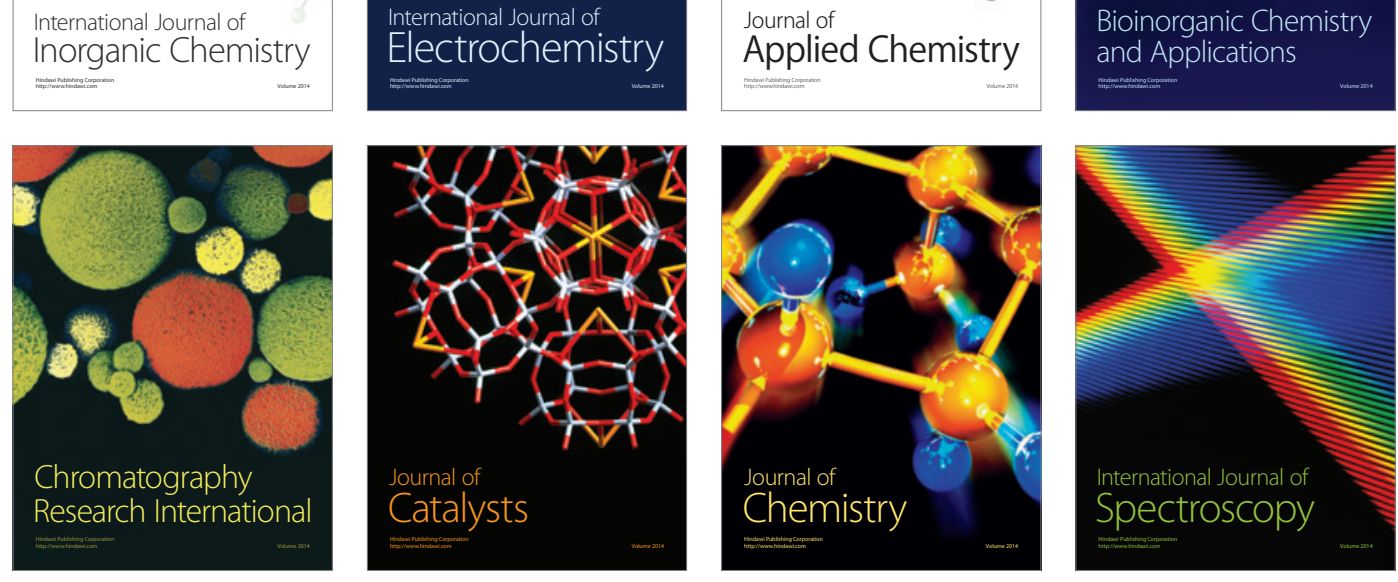